\title{
CODE DEVELOPMENT FOR NEXT-GENERATION HIGH-INTENSITY LARGE ACCEPTANCE FRAGMENT SEPARATORS
}

\author{
B. Erdelyi \# , L. Bandura, NIU, DeKalb, IL, 60115 and ANL, Argonne, IL 60439, U.S.A. \\ J. Nolen, S. Manikonda, ANL, Argonne, IL 60439, U.S.A.
}

\begin{abstract}
A suite of nuclear and atomic physics codes for computing a variety of quantities relevant for heavy-ions over a wide energy range passing through material were implemented in the DA-based beam optics code COSY Infinity. As extensions to COSY we also developed a limited fission model, map computation for beam-material interactions, and a hybrid map-Monte-Carlo mode that allows relatively fast and accurate fragment separator design, end-to-end modeling and simulations, and performance optimization. Some results are briefly mentioned; details will be published elsewhere.
\end{abstract}

\section{INTRODUCTION}

Fragment separators are essential components of some exotic beam facilities. Several such facilities are in operation world-wide, and next-generation facilities are coming online or planned for the near future. The scope of the separators is to separate, purify, and transport the isotopes of choice to the experimental stations while discarding the primary beam and unwanted by-products of the reaction mechanisms involved in the production of the rare isotopes. Beside the magnets for bending and focusing the beams, energy degraders are needed for a successful separation. For more details, we refer to [1]. The codes employed to design, simulate, and improve performance of fragment separators in an automated and integrated way are not readily available. Such codes need to be able to handle the optics to high order, including accurate and efficient treatment of fringe fields and measured fields. The atomic and nuclear processes need to be taken into account: energy loss in beam-material interactions, charge state distributions of heavy ions, straggling due to multiple collisions, production cross section for projectile fragmentation and fission, etc. The codes need to handle both aspects of the problem, optical and nuclear, in an integrated way. Moreover, the option to use numerical optimizers at the language level should be an essential feature.

Examples of codes used in the process of designing and simulating fragment separators are LISE++ [2] and MOCADI [3]. Our approach, that fulfills the above wish list, is to take a beam optics code that has all the necessary optics in it and the option of using numerical optimization, a suite of nuclear physics codes that do some aspect of the wish list very well, and come up with a framework that allows the use of all the above in an integrated way. In the following sections the existing codes that comprise the framework are discussed briefly,

"erdelyi@anl.gov

05 Beam Dynamics and Electromagnetic Fields followed by the detailing of the code development and concluding with some results.

\section{EXISTING CODES}

The following codes have been used to build the framework: COSY Infinity [4], ATIMA [5], GLOBAL [6], EPAX [7], and MCNPX [8]. A short description of these codes follows.

\section{COSY INFINITY}

COSY Infinity is an arbitrary order beam dynamics simulation and analysis code. It allows the study of accelerator lattices, spectrographs, beam lines, electron microscopes, fragment separators, and many other devices. It can determine high-order maps of combinations of particle optical elements of arbitrary field configurations. The elements can either be based on a large library of existing elements with realistic field configurations including fringe fields, or described in detail by measured data. COSY has its own object oriented programming language, and a differential algebra based computation engine. It includes optimization commands at the language level. Its features make it the ideal code for precision modeling, design, and optimization of fragment separators for next-generation exotic beam facilities.

The one component that needs to be included in COSY is the algorithms necessary to follow the distribution of charged particles through matter, which are necessary for isotope production, separation, and range bunching. Although for different reasons (related to muon cooling), COSY already has been extended to be able to track particles through matter for specific applications.

\section{ATIMA, GLOBAL, EPAX, and MCNPX}

ATIMA is a program developed at GSI, Germany, which calculates various physical quantities characterizing the slowing-down of protons and heavy ions in matter for specific kinetic energies ranging from 1 $\mathrm{keV} / \mathrm{u}$ to $450 \mathrm{GeV} / \mathrm{u}$ such as stopping power, energy loss, energy-loss straggling, angular straggling, range, range straggling, and several beam parameters (magnetic rigidity, time-of-flight, velocity, etc.).

The code uses different theories in different energy regimes and it includes a variety of corrections relevant for heavy ions. There is a variant of the code that uses precalculated values of the relevant quantities and local interpolations by splines. Although the resulting database is relatively large, it is a fast, accurate, and efficient way to obtain the energy loss and straggling values needed.

GLOBAL is a program developed at Berkeley, which calculates atomic charge-changing cross sections, charge- 
state evolutions, and equilibrium-charge-state distributions for relativistic heavy ions carrying up to 28 electrons during their penetration through matter. We use the code in the charge-state evolution mode.

EPAX assists in estimating production cross sections for projectile fragmentation reaction products, using a parametrization based on experimental data from highenergy reactions. It features a global fit using a simple functional form, hence it is really fast.

MCNPX is a general-purpose Monte Carlo radiation transport code for modeling the interaction of radiation with everything. A recent version that includes heavy-ion transport and a sophisticated fission model is used indirectly by our framework that will be described in the next section.

\section{CODE DEVELOPMENT}

We embarked on a code development program that had the purpose to provide a framework for end-to-end fragment separator simulations, including high order aberrations and accurate treatment of beam-material interactions. Also, fragmentation and fission crosssections and charge state evolution needed to be taken into account. Our choice was to take a state-of-the-art charged particle dynamics simulation code and implement and extend it to contain the new features needed for the above mentioned simulations. To this end, the functionalities of a suite of nuclear physics codes have been made an integral part of COSY Infinity.

COSY allows pretty straightforward addition of a new element to its already existing extensive list of dipoles, multipoles, cavities, both electric and magnetic, as well as elements for glass optics. A very important feature of the code is the existence of fitting procedures at the language level. With a choice of different optimizers the optimization problem can be cast in terms of the usual loops (FIT-ENDFIT).

We chose to include ATIMA (the spline-based version) for calculation of energy loss functions, and the various stragglings. We used EPAX 2.1 for calculation of fragmentation cross-sections. We implemented GLOBAL (and fixed some bugs to run under the widespread GNU compiler) for calculation of charge state evolutions. All of these stand-alone codes are now available as intrinsic function calls in COSY.

Full fission models tend to be overly complicated for our purposes. To this end we implemented a limited fission model (both cross-sections and kinematics) based on polynomial parametrizations of MCNPX-generated data.

The code runs in two modes: map mode and hybrid map-Monte-Carlo mode. In the map mode all deterministic effects are included in the map, and optimization can be done on the map real fast. In some cases, such as separation purity, for evaluating the performance of the whole system the hybrid mode is used.

\section{Fission model}

The most important quantities needed for the design and simulation of fragment separators are the number of fission products resulting from a given primary uranium beam of certain energy and the phase space distribution of these ions. Obviously, the angular and energy distribution is the interesting one since the spatial distribution follows the distribution of the primary beam, which is typically point-like.

We produced a database of cross sections for each isotope and the values for different incident energies were interpolated by polynomials. This is a fast and accurate way of estimating the cross sections and in the future the databases can be easily updated with the improvement of fission models.

\section{Wedge Map}

The deterministic effects can be included in a transfer map, much the same way as one computes a transfer map for a dipole or a quadrupole. In this sense, the energy degrader (wedge, absorber, target, etc.) can be viewed just as another optical element.

The deterministic effects are the average energy loss, and its dependence on the wedge's shape and a particle's initial conditions. For more details, we refer to [9]. By the usual composition methods the transfer map of the whole system can be computed to high orders and fast numerical optimization becomes possible in this approach. An example where this method can be applied is the optimization of the mass resolving power of the fragment separator as a function of wedge thickness.

\section{Monte-Carlo Absorber}

When the optical effects must be folded with the atomic and nuclear effects going on in the target and energy degraders, the map approach is not sufficient. The stochastic effects (charge state changing, angular straggling, energy straggling) and fragmentation/fission must be included in the Monte-Carlo approach. To this end, the MC absorber is sliced up into thin pieces in which we assume at most one nuclear reaction. We drift the existing particles to the end of the slice, apply the stochastic effects, determine how many new particles need to be created and what kind, and how many parent particles need to be deleted. After creation of the new particles, taking into account the parent phase space distribution, the reaction kinematics are applied (Fireball method in case of fragmentation, interpolation in case of fission), followed by the next slice. Of course, the number of slices is varied until convergence is achieved. From practice, we usually take one slice for every $5 \%$ of the range of the primary beam. This approach is analogous with the numerical approximation of integrals by summing up areas where the function value is assumed locally constant. It is also the most time consuming part of the code, especially in the target. 


\section{Hybrid Map-MC Running Mode}

For end-to-end simulations of fragment separators we developed a hybrid map-MC running mode. This consists of tracking a huge number of particles through the separator and applying the map in the regions where beam-material interactions are absent and the $\mathrm{MC}$ absorber elsewhere. This is the most efficient way of running large numbers of particles needed to study separation purity and background distributions. The achieved performance is tracking all particles generated by the incidence of $10^{8}-10^{9}$ (depending on the case) primary beam particles on a target over the length of a two-stage separator on a typical one-processor Linux box in about a day. The details vary significantly as a function of the primary beam type and energy, the target material and thickness, the selected isotope, the slit settings, and the type and thickness of energy degraders.

\section{RESULTS}

Due to lack of space, here we include just samples of some representative results. In Figure 1 the phase space distribution of ${ }^{132} \mathrm{Sn}$ created by the fission of $200 \mathrm{MeV} / \mathrm{u}$ ${ }^{238} \mathrm{U}$ is presented. Our simplified model is able to accurately reproduce the number of ions as well as their distribution.

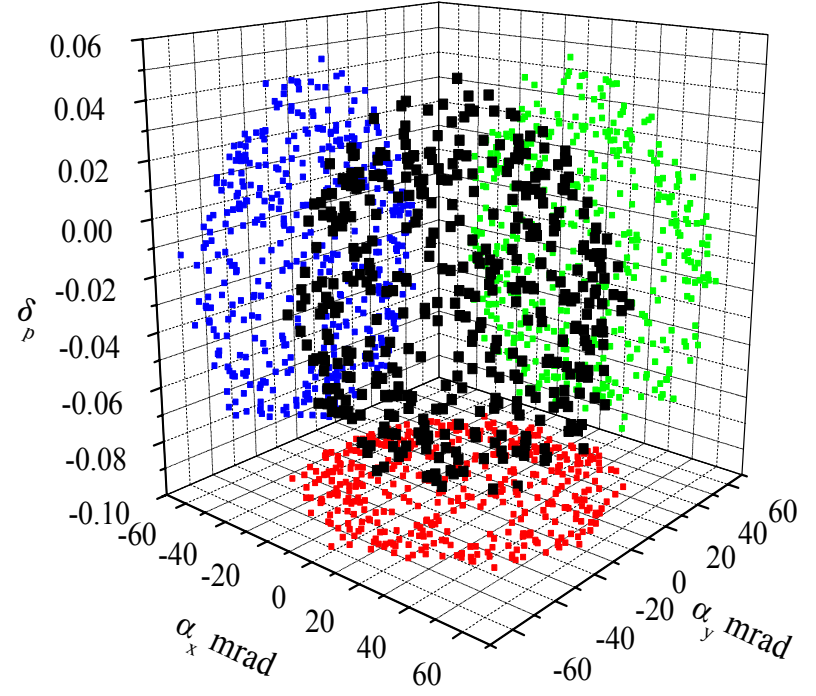

Figure 1: An example for the distribution of a fission product: ${ }^{132} \mathrm{Sn}$ obtained from ${ }^{238} \mathrm{U}$ on Li target.

An example of the power of the map approach that includes the wedge is shown in Figure 2. The optimization, which can be done in minutes for this example, shows that there is a broad maximum for the resolving power around $40 \%$ of the range, and interestingly the optimum thickness is pushed to slightly larger values by the residual aberrations.

We illustrate the separation purity with Figure 3: a ${ }^{124} \mathrm{Xe}$ beam of $1500 \mathrm{MeV} / \mathrm{n}$ is used to produce ${ }^{100} \mathrm{Sn}$ in a Li target. A two-stage separator is used to separate the tin isotopes and the picture shows the isotopes that go through the final slit. As shown in Figure 3, the separated beam is an almost pure ${ }^{100} \mathrm{Sn}$ beam.

05 Beam Dynamics and Electromagnetic Fields

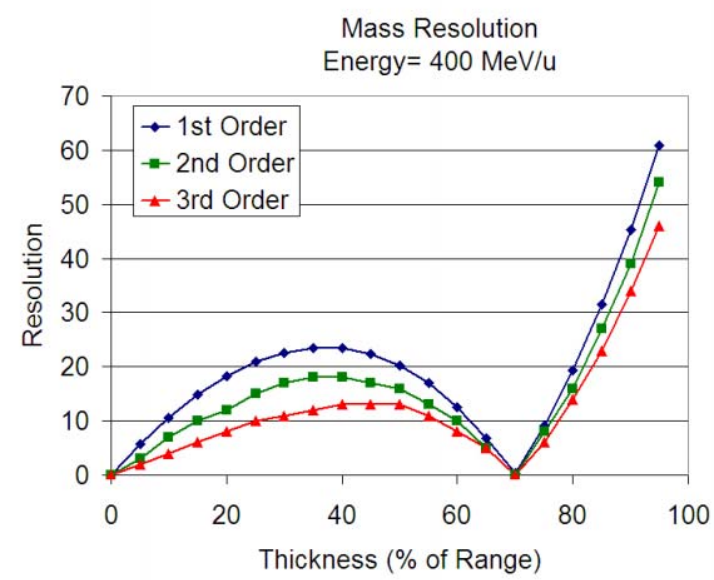

Figure 2: An example for the optimization of the mass resolving power as a function of thickness at various orders of evaluation of the resolution.

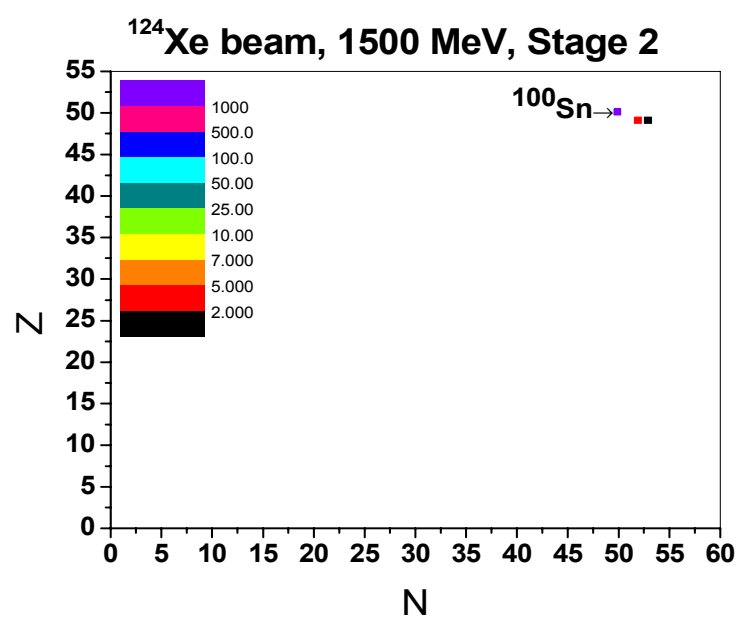

Figure 3: An example for the separation purity (events in arbitrary units) of a two-stage separator: ${ }^{100} \mathrm{Sn}$ is separated from all primary beam and background ions except a couple of weak nearby isotopes.

\section{ACKNOWLEDGEMENTS}

We would like to thank I. Gomes for providing the MCNPX data we used to generate our fission database, T. Barlow for work on the COSY extensions, and M. Berz's group on work related to wedge maps. This work was supported by the U.S. Department of Energy, Office of Nuclear Physics, under Contract No. DE-AC02$06 \mathrm{CH} 11357$.

\section{REFERENCES}

[1] B. Erdelyi et al, Phys. Rev. ST-AB, accepted (2007).

[2] http://groups.nscl.msu.edu/lise/lise.html

[3] http://www-linux.gsi.de/ weick/mocadi/

[4] http://bt.pa.msu.edu/index_files/cosy.htm

[5] http://www-linux.gsi.de/ - weick/atima/

[6] http://www-linux.gsi.de/ weick/charge_states/

[7] http://www-w2k.gsi.de/hellstr/asp/gsi/epaxv21m.asp

[8] http://mcnpx.lanl.gov/

[9] L. Bandura et al., these proceedings (2007).

D01 Beam Optics - Lattices, Correction Schemes, Transport 